\title{
A Short Supply Chain to Guarantee
}

\section{Wood-Chip Quality}

\author{
Roberto Deboli ${ }^{1}$, Massimiliano Ruggeri \\ Institute of Agricultural and Earthmoving Machines - \\ Italian National Research Council, Italy \\ Angela Calvo \\ Department of Agriculture, Torino, Italy
}

\begin{abstract}
Copyright (C) 2014 Roberto Deboli et al. This is an open access article distributed under the Creative Commons Attribution License, which permits unrestricted use, distribution, and reproduction in any medium, provided the original work is properly cited.
\end{abstract}

\begin{abstract}
It is important to know the origin of the wood chip and to verify the whole transport chain, from the forest to the processing plant. This can be done using electronic customized devices to monitor the entire process: these technologies, already used in other fields, are based on the transmission of GPS data via GPRS-UMTS-HSDPA connection. In the wood chip supply chain application they must be adapted to the context, properly tailored to interface with the electronics onboard the machine and to generate usable arrays of data, including performance and quality.

In this work the entire chain is controlled by means of a novel electronic architecture onboard the machine, interfaced with a communication system connected to a central server acting as a supervisor. All the machines are equipped with a GPS receiver, a data communication module and a CAN (SAE J1939) interface: machines data are sent to a central server, through an internet connection, and collected in a database ready for web services. A communication protocol has been designed to optimize data transfer and to minimize the cost. To increase the product value, an automated integral sensor (capacitive type) for wood chip humidity determination was implemented. With the fleet management system installed on the machine, cruise controls data (using a GPS system) are acquired, other than all the machine states (through CAN line inside the machine) and wood humidity.
\end{abstract}


The data base (MySQL standard) permits, moreover, to the system administrator and to the user to manage all these information. Another important result is the web access that permits to monitor the fleet situation (route, engine status, time of journey, climatic conditions) at any moment with a screenshot. The capacitive sensor for humidity measurements gives good results, especially in the case of chips with dimensions comprised between 34 and $64 \mathrm{~mm}$ and an initial humidity (on wet basis) of $45 \%$.

Keywords: wood-chip, wood quality, wood supply-chain

\section{Introduction}

In Italy the net usable area for energetic purpose is around 6 million hectares but many woods are located in areas difficult to get at (e.g. by physical constraints like steep slopes or wet soil, by availability of harvesting equipment or labor, by tiny volumes coming from fragmented supply areas or by deficiency of forestry roads). The mechanization level for their exploitation is definitely low: as a consequence, the production costs are high, at the boundaries of the profit (Allen et al., 1998; Bjornstad, 2004, Monarca et al, 2011, Colantoni et al. 2013).

Many authors considered this problem; some of them (Matthies et al., 2007; Frombo et al., 2009) suggested environmental decision support systems based on GIS methodologies, as well as mathematical models to locate the intervention area and to dimension the mechanized chain, to determine the wood fuel harvest and store criteria in function of the energy to be produced. The same authors did not neglect economic and social aspects as well.

The wood fuel is a poor and bulky product: for this reason the cost of transport risk to be remarkably high for the final cost of the merchandise (Erikson and Bjorheden in 1989 found that transportation cost constituted the most essential part of total cost for forest fuel). Moreover, the environmental advantages of the biomass to produce energy ( $\mathrm{CO} 2$ balance) risk to be mostly reduced when the transport distance from the forest to the energy plants become higher (Timmons and Mejia, 2010).

Woodchips becomes so competitive, because they are easier to transport: a truck which can transport 65 stacked cubic meters of log, can transport 80 bulk cubic meters of chips (Gronalt and Rauch, 2007). Asikainen et al. (2001) stated that for distances up to about $50 \mathrm{~km}$ material can be transported in loose form, at longer distances chipping before transport is competitive.

The advantage of woodchip depends also on other local factors, such as the raw material availability, facilities (store chip terminals, chippers size) and the energy plant power: in fact, a power plant of 1 MWe consumes around $15 \cdot 106 \mathrm{~kg} /$ year of woodchip, corresponding to an exploitable area of 550 hectares.

When the potential supply volume for forest fuels has been evaluated, another important aspect to be considered is where to chip (figure 1): directly in forest or in local terminals (using mobile chippers) or in a central industrial hub which provides forest fuels to the energy plant or to other end users, using a big chipper? 


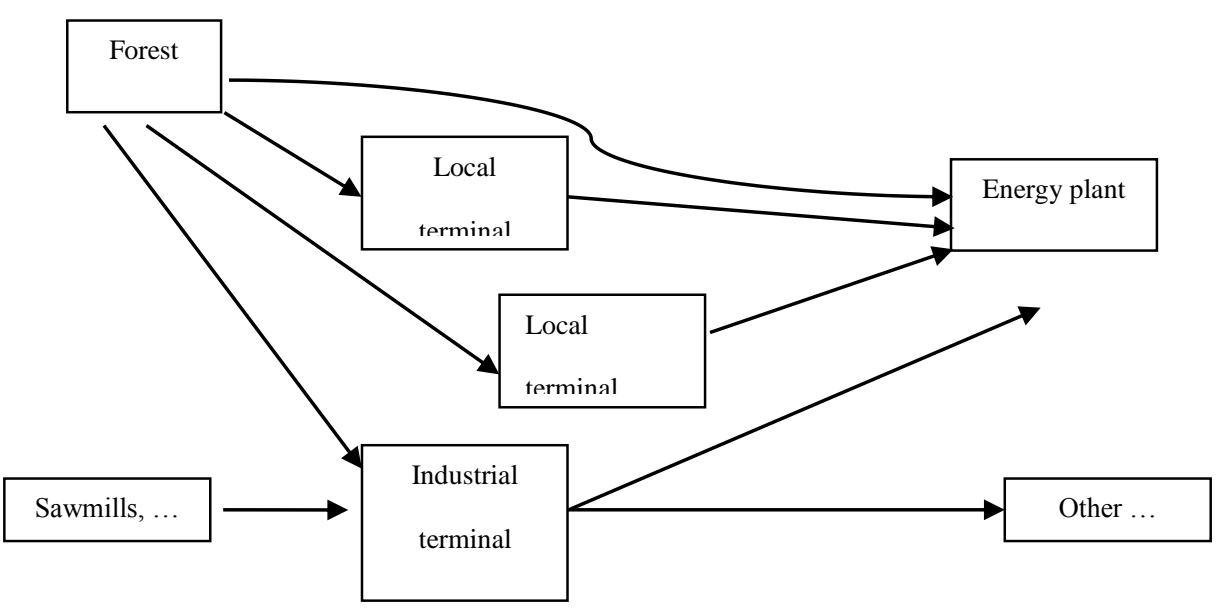

Figure 1. Different solutions for an energy plant wood chip fuel supply

Different chipping points in the chain and different weight to volume ratios of material to be transported can be combined in different ways to form a suitable supply chain; local terminals, as well as industrial terminals, can be used to supply one or more plants and can be used for storing and chipping if plants cannot do that (Gronalt and Rauch, 2007). Nevertheless the industrial terminal may be connected to other timber plants, such as sawmills or papermills, where wooden by-products of poor quality may be present. Local terminals, indeed, must be designed to guarantee fuel to the energy plant in each moment. The organization and supply of the logistics depends mainly on three factors: provided space, given infrastructure and season / time. In comparison to highly standardized products (e. g. in the automotive industry) long-term planning for the supply of qualitative comparable woodchip products is hard to realize. Local terminals for woody biomass are a possibility to overcome these problems by bundling, storing and converting woody biomass to develop and organize local woodchip supply.

Material flow is a focal point for the forest fuel supply network, because it is important to know the origin of the wood chip and to verify the whole transport chain, from the forest to the processing plant. This can be easily done in the short wood chain system (Sikanena et al., 2005), using electronic customized devices to monitor the entire process: these technologies, already used in other fields, are based on the transmission of GPS data via GPRS-UMTS-HSDPA connection.

In this specific application they must be adapted to the context, properly tailored to interface with the electronics onboard the machine and to generate usable arrays of data, including performance and quality (Fantuzzi et al., 2006).

In this work the entire chain is controlled by means of a novel electronic architecture onboard the machine, interfaced with a communication system connected to a central server acting as a supervisor. All the machines are equipped with a GPS receiver, a data communication module and a CAN (SAE J1939) interface: machines data are sent to a central server, for message server, database and web services. A communication protocol has been designed to optimize data 
transfer and to minimize their cost (Ravenaz et al., 2010). To increase the product value, an automated integral sensor (capacitive type) for wood chip humidity determination was also implemented.

\section{Material and methods}

The continuous monitoring of wood chipper (working hours, engine speed, wood chipper speed, active engine torque, service operations, etc.) is useful to certify the relative energy consumption (fuel liters vs. wood chip amount) related to wood chip produced and delivered to customers; moreover the same data are useful in order to prove the overall cost of the wood chip production and its positive energy balance. In order to obtain this set of heterogeneous data a set of sensors was used and installed on the machine, all connected to both its mechanical and electrical parts, in order to directly acquire information related to physical measurements. Moreover, wood chipper and the truck used to move the wood chip have been equipped with electronic embedded systems, to get the information of the geo-referenced vehicle position. Another important parameter to collect for the wood chip quality is the wood humidity, because more is humid the wood, more is the energy consumed to evaporate the water inside it. Actually to calculate wood-chip moisture there are moisture meters of different types: some use the characteristics of polarity of water molecules, other utilize magnetic characteristics of hydrogen or its capacity to absorb electromagnetic radiations. Resistive meters are based on the capacity of moisture to reduce the electric resistance, while other use the capacitive measurement system, based on the measure of voltage of an electric field passing through a moist mean, exploiting the inversely proportional ratio between moisture and voltage. This type of meters, called capacitors, are present in some models, inserting and extracting them in wood-chips by hands; it requires a remarkable use of time and human resources, so even some quantity of money. To solve this disadvantages, the C.N.R. Imamoter Institute planned and realized a wood-chip moisture meter designed to be directly placed in the woodchipper, in order to measure moisture continuously.

For the explained reason, the main parts of the job were:

1. An ad hoc wood chip chain fleet management system;

2. A capacitive wood-chip moisture meter to continuously acquire the wood chip humidity in the wood chipper.

\subsection{Fleet management system for woodchip supply chain}

Today the widely used application of fleet management is related to alarm and to fleet control systems, in order to monitor the vehicle position, the road, the travel time, the fuel consumption, the speed and other data related to the vehicle and to the driver performance. In this way the goods traceability is available both in real time and historically, thanks to the data post processing based on the information database. 
For the wood chip chain control, after a detailed analysis of the available products, it was established that it was impossible to adopt existing products on fleet management, for the connection with both the CAN SAE J1939 to control the operator's work and the private wood chip machine through a private CAN line. The fleet management systems actually available are able to calculate and to send data with GPS position and vehicle speed (calculated from the GPS positions acquired at different time). It is unusual to find a fleet management system with a CAN line, almost impossible to find one equipped with the CAN SAE J1939 protocol, absolutely impossible to find something customizable for the woodchip chain control.

None system available in the market can be freely modified, for example to collect data from a wood chipper machine. Also is a customization were permitted, the basic problem is the variable length of data to be sent.

For these reasons a new system was designed, to collect and to manage both GPS information and input data from the truck powertrain network and from the private wood chipper network through a gateway. This new system uses a secondary CAN network for the wood chipper and generates CAN SAE J1939 personal messages, properly mapped in the fleet management database inside the fleet management ECU (Electronic Control Unit) on board. Data collected from the sensors and from the CAN lines are properly packed in a User Datagram Protocol (UDP) message and sent to a remote server via a socket periodically open in internet (with a programmable timing, starting from the smallest 10 seconds period).

This system is now adaptable to any wood chipper or to any working machine, simply changing the message map periodically downloadable on the fleet management ECU through the server.

The main components of the ECU fleet management system in the vehicle are:

- a microcontroller ECU embedded, equipped with serial lines and an High Speed CAN Controller, using the SAE J1939 protocol at higher level, - a GPS receiver, to georeference the wood chipper position,

- a GPRS/UMTS/3G modem, for data communication through internet via a mobile network structure.

In the remote company server or in the cloud, indeed, the components are:

- a data exchange server, to manage the sockets and the messages from the yard machines,

- a customized database, for customers' needs,

- a query system managed by a web server to elaborate, visualize, analyze and map data collected in the database. 


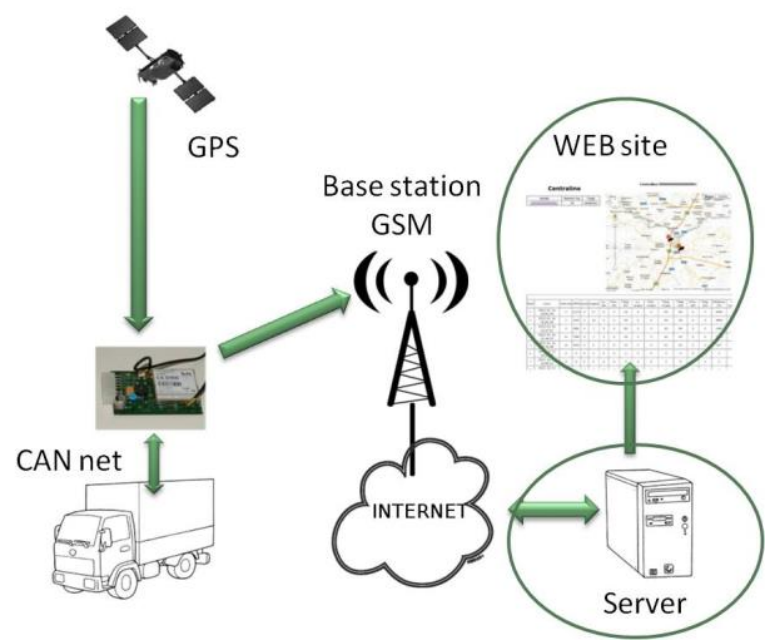

Figure 2. Fleet management system diagram for woodchip supply chain

From the data management point of view, it can be highlighted that all data (field information, 32 bits GPS related, messages from the truck engine, data from the digital tachograpf, data from the drum chipper related to the machine status and the wood humidity) are collected by the microcontroller based ECU. Data are properly packed and periodically sent through a socket directly open on the central server, using UDP messages, in order to minimize the protocol overhead. In fact, data transmission costs are proportional to the amount of bytes sent on the wireless link in the mobile phones infrastructure, unless the bits are information related or simply protocol overhead. With its $1,5 \mathrm{~kb}$ structure, the TCP/IP packets does not fit the 130 bytes of data to be periodically sent by the application: for this reason the UDP protocol was used. Also if it lacks of the handshaking procedures (with the consequence of a lower safety level), the UDP offers a perfect fit on dimension of the messages (with lower transmission costs) and improves data receiving, directly performed by the server that can send data requests to the embedded ECU in the vehicle.

The "pay by traffic" rates are convenient if a special attention is posed on the data traffic amount, especially in roaming condition. The foreseen and proved monthly average cost for the machine data transmission, considering 12 working hours/7 days is around 3 euros/month. A central server was entirely designed and developed to test the communication feasibility and robustness at the IMAMOTER Institute of Ferrara. The server is properly running and it is collecting data from the wood chip chain.

\subsection{System for the continuous woodchip humidity determination}

The real time knowledge of woodchip moisture let the operator to know the suitable place for the drying and the possible anomalies in the fuel.

The moisture material content MC is typically defined on a wet basis as [21]: 


$$
M C=\frac{m_{w e t}-m_{d r y}}{m_{w e t}} \cdot 100
$$

where $\mathrm{m}$ denotes the mass of the sample.

The capacitive wood chip moisture meter has been realized to work in the wood chipper, to let the continuous measurement of the chip moisture, more times in a minute, immediately after the chips output from the cutting device. The moisture meter consists of two copper sheets head on (30 x $40 \mathrm{~cm}$ each), $15 \mathrm{~cm}$ far and screwed on a wooden structure. The two sheets are connected to a circuit which is linked to a meter voltage. The circuit (figure 3 ) is in a stable configuration (which produces a square wave) with a frequency inversely proportional to the capacity value and, as a consequence, to the moisture.

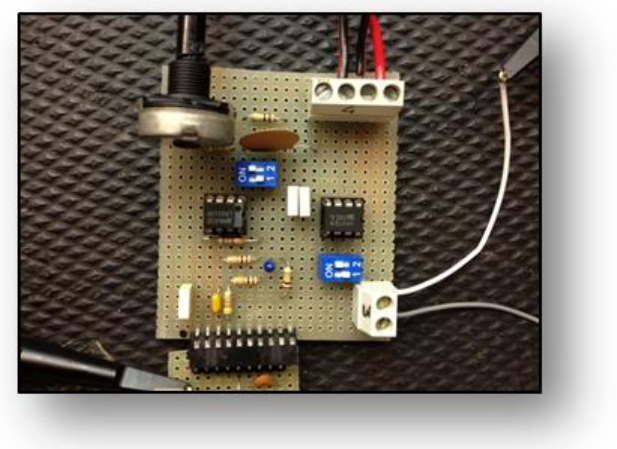

Figure 3. Moisture meter circuit

The output frequency is converted in a voltage by a frequency/voltage converter. A precision circuit is present to condition the output electric signal. The prototype of capacitor was calibrated to give a correct moisture value to each voltage; during the executing of the tests for the calibration, some modification to the structure and to the circuit were done to make the instrument more precise. Many test were conducted, starting from different input moisture values and decreasing them to fit the voltage to the chip moisture content. Moreover, chips samples were then divided in dimension classes (35-64 mm, 16-34 mm and less than $16 \mathrm{~mm}$ ) to understand how the electric field inside the capacitor could be influenced by the ratio wood/air and by the chips position between the copper sheets.

\section{Results}

At the moment from the CAN net it is possible to manage about fifty information, some of them specific of the machine (driveline engaged, current gear, park brake, oil level, temperature and pressure, ..), others related to the engaged work (RPM, fuel level, ...), velocity, distance from the target point, other than the GPS position. 


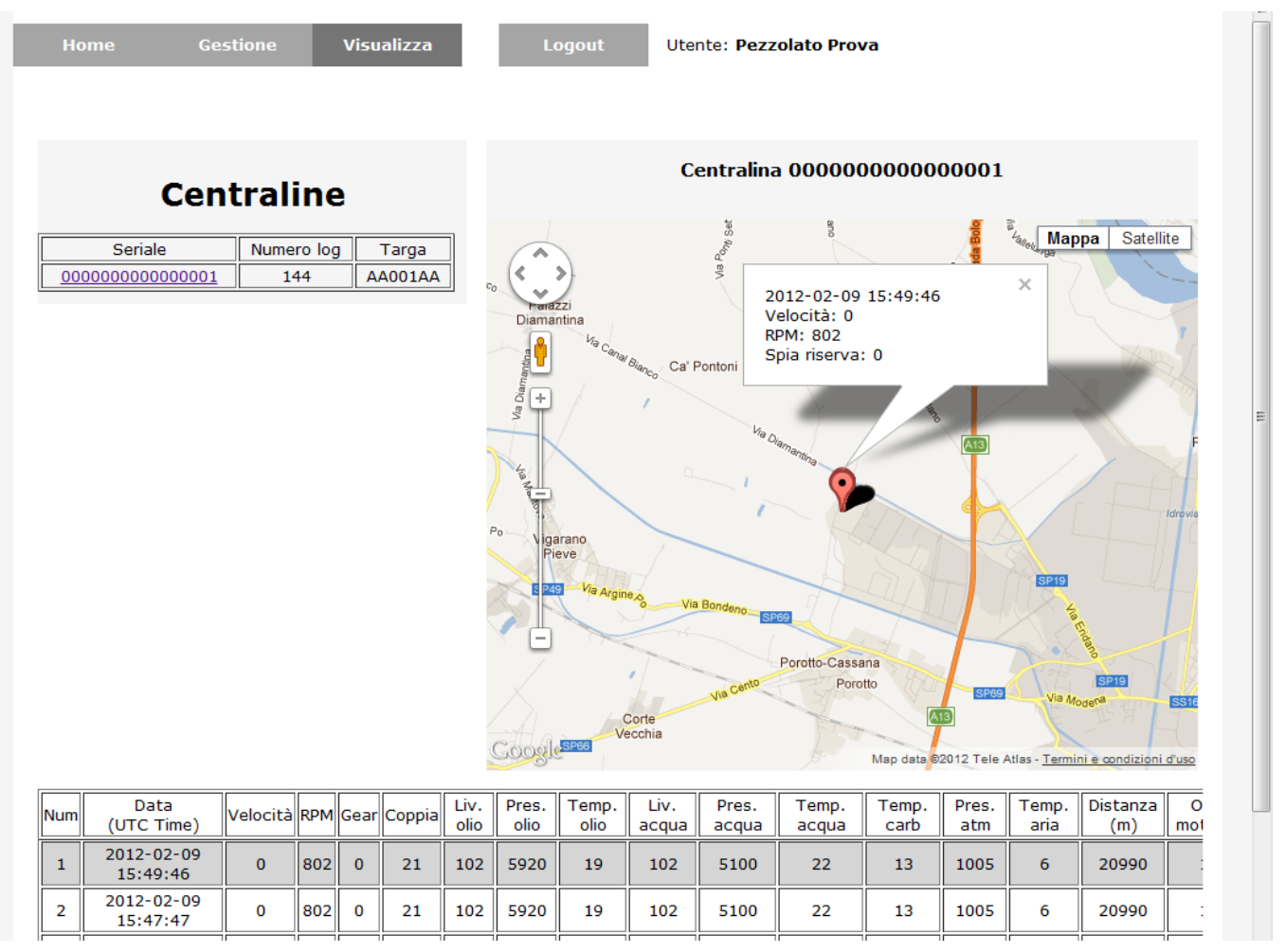

Figure 4. Example of information screenshot

The database (MySQL standard) permits to the different users type (administrator, system manager, user) to access to the stored information, both at local level and in the web site and, when they are authorized, to manage them.

Concerning the wood chip moisture meter, results show that, as expected, with the decrease of the moisture, the voltage increases and the two quantities are inversely proportional. Some differences in the results have been observed concerning the chips size (figure 5): in the wood-chips with large size there is a considerable quantity of air that increase the values of voltage, whereas wood-chip with small size is often associated with sawdust that, on the contrary, reduce the voltage values; these observations are present also in all the existing capacitive measurement instruments, as proof of the fact that the prototype operate correctly. Moreover, best results were detected with an initial humidity (on wet basis) of $45 \%$. 


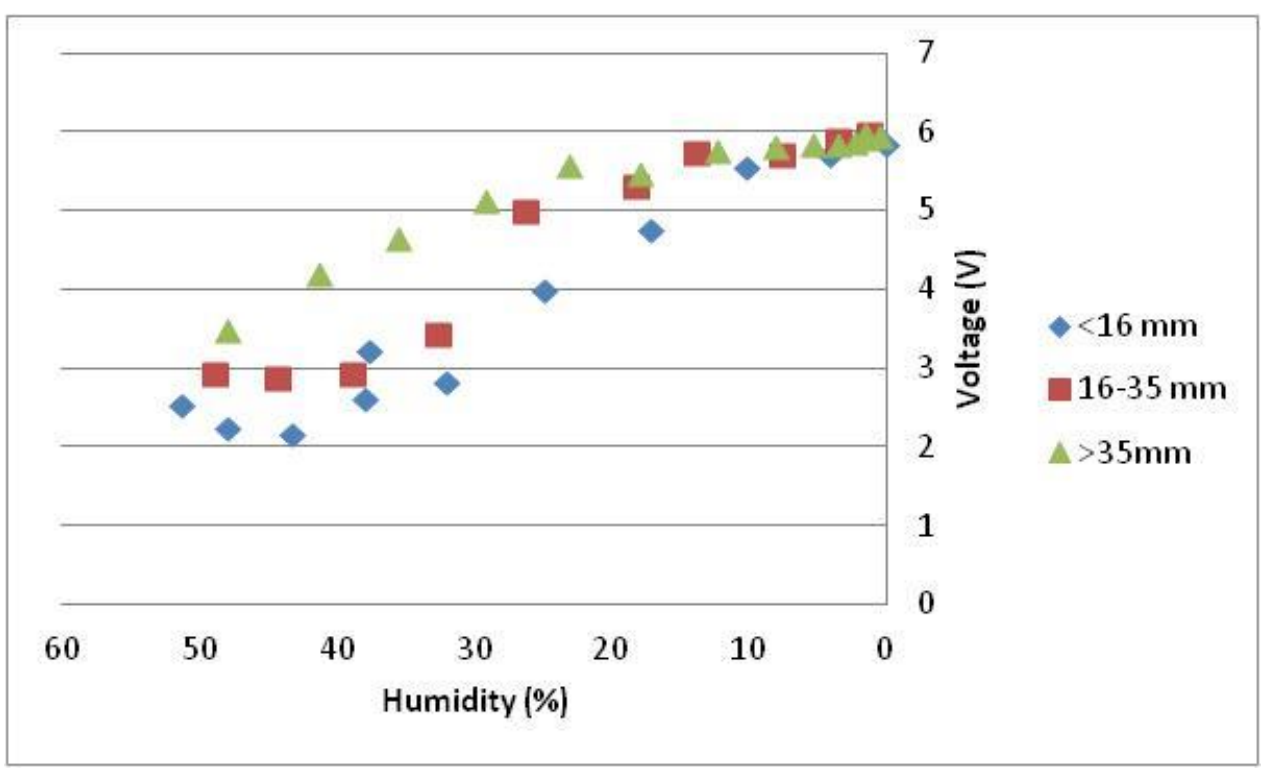

Figure 5. Wood chips (with different chips size) voltage measured at different moisture in the moisture meter

Acknowledgements. The project was funded by the Piemonte Region.

\section{References}

[1] D.H. Ackley, G.E. Hinton and T.J. Sejnowski, A learning algorithm for Boltzmann machine, Cognitive Science, 9 (1985), 147 - 169.

[2] F.L. Crane, H. Low, P. Navas, I.L. Sun, Control of cell growth by plasma membrane NADH oxidation, Pure and Applied Chemical Sciences, 1 (2013), 31 42. http://dx.doi.org/10.12988/pacs.2013.3310

[3] D.O. Hebb, The Organization of Behavior, Wiley, New York, 1949.

[4] Allen, J., M., Browne, A., Hunter, J., Boyd and H. Palmer. 1998. Logistics management and costs of biomass fuel supply. International Journal of Physical Distribution and Logistics Management 28(6): 1-10.

[5] Asikainen, A. 2001. Design of supply chains for forest fuels. In Supply chain management for paper and timber industries, eds. K. Sjöström, 179-190. Finland.

[6] Asikainen, A., T., Ranta and J., Laitila. 2001. Large-scale forest fuel procurement. In Proc. Woody biomass as an energy source-challenges in Europe: EFI, eds. P. Pelkonen, P. Hakkila, T. Karjalainen and B. Schlamadinger, 39: 73-8.

[7] Bjornstad, E. 2004. An engineering economics approach to the estimation of forest fuel supply in Nord-Trondelag country, Norway. Journal of Forest Economics 10: 161-188. 
[8] Erikson, L. and R., Bjorheden. 1989. Optimal storing, transport and processing for a forest-fuel supplier. European Journal of Operations Research 1(43): 26-33.

[9] Fantuzzi, C., M., Ruggeri, S., Marzani and C., Secchi. 2006. A Distributed Embedded Control System for Agricultural Machines. In: Proc. 4th International IEEE Conference on Industrial Informatics: 898-903, INDIN'06, Singapore, 16-18 August.

[10] Frombo, F., R., Minciardi, M., Robba, F., Rosso and R., Sacile. 2009. Planning woody biomass logistics for energy production: a strategic decision model. Biomass \& Bioenergy. 33(3), 372-383.

[11] Gronalt, M. and P., Rauch. 2007. Designing a regional forest fuel supply network. Biomass \& Bioenergy 31(6): 393-402.

[12] Matthies, M., C., Giupponi and B., Ostendorf. 2007. Environmental decision support systems; current issues, methods and tools. Environmental Modelling and Software 22: 123-127.

[13] Revenaz, A., M., Ruggeri and M., Martelli. 2010. Wireless Communication Protocol for Agricultural Machines Synchronization and Fleet Management In: Proc. 2010 IEEE International Symposium on Industrial Electronics (1) 3498-3504, Bari, 4-7 July.

[14] Timmons, D. and C. V., Mejia. 2010. Biomass energy from wood chips: Diesel fuel dependence? Biomass \& Bioenergy 34(9): 1419-1425.

[15] Sikanena L., A., Asikainen and M., Lehikoinen. 2005. Transport control of forest fuels by fleet manager, mobile terminals and GPS. Biomass and Bioenergy. 28(2): 183-191.

[16] D. Monarca, M. Cecchini, A. Colantoni (2011). Plant for the production of chips and pellet: technical and economic aspects of a case study in the central Italy. In: (a cura di): (C) Springer-Verlag Berlin Heidelberg 2011, In: Computational Science and Its Applications. Santander (Spain), June 20-23, 2011. . vol. Part IV, p. 296-306, ISBN: 978-3-642-21897-2, Spain, June 20-23, 2011., doi: 10.1700/978-3-642-21898-9.

[17] Colantoni A, Allegrini E, Recanatesi F, Romagnoli M, Biondi P, Boubaker K (2013). Mathematical Analysis of Gasification Process Using Boubaker Polynomials Expansion Scheme. In: The 13th International Conference on Computational Science and Its Applications. LECTURE NOTES IN COMPUTER SCIENCE, vol. 7972, p. 288-298, ISBN: 978-3-642-39643-4, ISSN: 0302-9743, Ho Chi Minh City, Vietnam, June 24-27, 2013, doi: 10.1007/978-3-642-39643-4_22.

[18] A. Fuchs, H. Zangl, G. Holler, G. Brasseur: "Design and Analysis of a Capacitive Moisture Sensor for

[19] Municipal Solid Waste". Measurement Science and Technology, vol. 19, 2008.

[20] Marucci, A., Carlini, M., Castellucci, S., Cappuccini, A. "Energy efficiency of a greenhouse for the conservation of forestry biodiversity". Mathematical Problems in Engineering, 2013.

\section{Received: June 1, 2014}

